\title{
The Effectiveness of Autologous Serum and Single-dose Oral Doxycycline in the Treatment of Acne Vulgaris: A Pilot Clinical Trial
}

\author{
Muhammad Nasrum Massi ${ }^{1 *}(\mathbb{D})$, Muhlis Yunus ${ }^{2}$, Anis Irawan Anwar ${ }^{2}$ (D), Khairuddin Djawad ${ }^{2}$, Siswanto Wahab ${ }^{2}$ (D), \\ Rahmawati Minhajat $^{3} \mathbb{D}$, Uleng Bahrun ${ }^{4} \mathbb{D}$, Ilhamjaya Patellongi $i^{5}$, Gemini Alam ${ }^{6} \mathbb{D}$, Ni Nyoman Sri Budayanti ${ }^{7}$ (D) \\ ${ }^{1}$ Department of Clinical Microbiology, Faculty of Medicine, Hasanuddin University, Makassar, Indonesia; ${ }^{2}$ Department of \\ Dermatology and Venereology, Faculty of Medicine, Hasanuddin University, Makassar, Indonesia; ${ }^{3}$ Department of Internal \\ Medicine, Faculty of Medicine, Hasanuddin University, Makassar, Indonesia; ${ }^{4}$ Department of Clinical Pathology, Faculty \\ of Medicine, Hasanuddin University, Makassar, Indonesia; ${ }^{5}$ Department of Physiology, Faculty of Medicine, Hasanuddin \\ University, Makassar, Indonesia; ${ }^{6}$ Department of Pharmacy, Faculty of Pharmacy, Hasanuddin University, Makassar, Indonesia; \\ ${ }^{7}$ Department of Clinical Microbiology, Faculty of Medicine, Udayana University, Denpasar, Bali, Indonesia
}

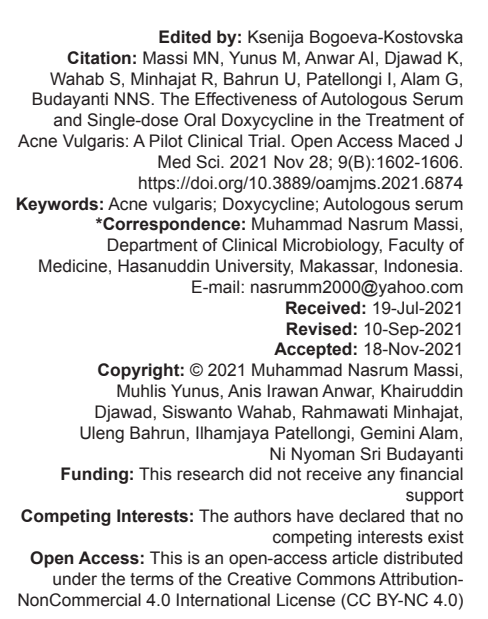

\section{Abstract}

BACKGROUND: Acne vulgaris is a chronic inflammatory disease that occurs with various factors, both intrinsic and extrinsic. Treatment of choice can vary from topical, systemic or a combination of both depending on the degree of severity. Recently, treatment using products from human blood has been widely studied, including in the field of dermatology as it has a unique composition.

AIM: The objective of the study was to assess the effectiveness of autologous serum and single-dose oral doxycycline in the treatment of acne vulgaris

METHODS: This clinical trial compared two groups which received autologous serum only and autologous serum combined with single dose of oral $200 \mathrm{mg}$ doxycycline, respectively. Photographic pictures and number of lesions (blackheads, papules, and pustules) were compared before and after application using Wilcoxon test.

RESULTS: A total of 20 participants participated in this study. Both treatment groups showed significant decrease in lesion count $(p<0.01)$. However, the group which received autologous serum and single dose of oral doxycycline showed superior improvement of inflammatory acne lesions.

CONCLUSION: Autologous serum and single dose of oral doxycycline was superior to autologous serum only in the treatment acne lesions.

\section{Introduction}

Acne vulgaris is a chronic inflammatory disease that occurs due to a variety of both intrinsic and extrinsic factors. Treatments vary from topical, systemic, or a combination of both depending on the degree of severity. Acne vulgaris is a disease of the pilosebaceous unit, the sebaceous glands are found throughout the human skin and has greater concentration on the face and scalp but absent on the palms of the hands and soles of the feet. These glands store sebum in the hair and carry it to the surface along the hair shaft [1]. Sebum provides lubrication and hydration and composed of triglycerides, free fatty acids, and aliphatic hydrocarbons [2]. Acne arises from the interaction of four major pathogenic processes: Hyperproliferation of sebaceous glands; hyperproliferation of keratinocytes; colonization of Propionibacterium acnes; and the presence of inflammation. These pathogenic factors influence each other and should not be approached individually. Although the interaction of pathogenic factors is known to cause acne, certain trigger factors can worsen or cause acne to appear. Examples of these trigger factors are premenstrual and cosmetic use [3]. Treatment can vary from topical, systemic or a combination of both depending on the degree of severity. Recently, treatment using products from human blood has been widely researched, including in the field of dermatology because of its unique composition [4].

Therapeutic modalities for the treatment of acne vulgaris include topical and systemic therapy. Topical therapy includes the use of antibiotics and Vitamin A derivatives while options of systemic therapy are oral antibiotics, Vitamin A derivatives and hormonal drugs. Based on the pathomechanism of acne vulgaris, the current therapeutic modality can be given through topical, systemic, or a combination of both [5]. However, 
in certain cases, such as hypersensitivity reactions, these drug preparations cannot be administered. These conditions include allergies and unwanted side effects [6]. This study aims to assess the effect of autologous serum derived after single-dose doxycycline administration and doxycycline in autologous serum in the treatment of acne vulgaris.

\section{Methods}

This was a pre- and post-treatment clinical trial in two treatment groups with each group consisted of ten people with acne vulgaris. All eligible subjects were randomly allocated into two groups: Those receiving autologous serum and those receiving oral doxycycline, respectively. Subjects were equally divided into two groups with one group receiving autologous serum and another receiving a combination of autologous serum and a single dose of oral $200 \mathrm{mg}$ doxycycline; both treatments were applied for 28 days. Males and females aged 15-35 years old with no recent or current acne therapy and not pregnant (for women) were eligible to participate in this study. This study had obtained approval from the local ethical board committee.

The autologous serum in the first group was made by drawing $50 \mathrm{cc}$ blood from the cubital vein which was then inserted into a tube without a coagulant. The tube was then centrifuged for 10-15 min at a speed of $3000 \mathrm{rpm}$ which separated the clear blood plasma from the blood. Meanwhile, the other group was given a single dose of oral $200 \mathrm{mg}$ doxycycline and, after $2 \mathrm{~h}$, had $50 \mathrm{cc}$ of venous blood drawn followed by the same centrifugation process. The serum obtained from the centrifuge process was put into a small bottle containing 1-2 cc for one application. The serum was stored in a refrigerator at $0^{\circ} \mathrm{C}$ and applied every night to areas of the face with acne lesions for 28 days. Before the serum was applied, patients were asked to wash their face without using soap or other cosmetic products. To see the changes that occur, photographic pictures and number of lesions (blackheads, papules, and pustules) were compared before and after application.

\section{Results}

A total of 20 subjects participated in this study. All participants were female and the average age in each group was 22.4 years and 24.6 years ( $p>0.05$ ).

In the autologous serum only group, an improvement of acne lesions was seen after 28 days (Figure 1). A decrease in lesions by $11.7 \%$, papules $15 \%$, and pustules $15.4 \%$ was seen after the administration (Table 1). Wilcoxon test showed that the number of blackheads, papules, and pustules decreased significantly after 28 days of treatment $(p<0.05)$.

Table 1: Post-treatment result of the autologous serum only group

\begin{tabular}{lll}
\hline Variable & D1- D28 & \\
\cline { 2 - 3 } & Median & p-value \\
\hline Decrease in number of blackheads (\%) & $60.2(11.7)$ & $<0.001$ \\
Decrease in number of papules $(\%)$ & $54.4(15.0)$ & $<0.001$ \\
Decrease in number of pustules (\%) & $78.9(15.4)$ & $<0.001$ \\
\hline${ }^{*}$ Significant if $\mathrm{p}<0.05$ (Wilcoxon test). &
\end{tabular}

In the autologous serum and doxycycline group, an improvement of blackhead lesions was seen after 28 days (Figure 2). A decrease in lesions by $18.8 \%$, papules $26.6 \%$, and pustules $56.0 \%$ was seen after the administration (Table 2). Wilcoxon test showed that the number of blackheads, papules, and pustules decreased significantly after 28 days of treatment $(p<0.05)$.

\section{Discussion}

In patients with acne vulgaris, hyperproliferation of keratinocytes that occur results in the accumulation of abnormal corneocyte desquamation on the sebaceous follicles along with lipids and monofilaments, resulting in the formation of blackheads. $P$. acne is a Gramnegative anaerobic pathogen colonizing the sebaceous follicles. In accordance to the pharmacokinetic properties of doxycycline, blood sampling is taken every $2 \mathrm{~h}$ after the administration of oral doxycycline $200 \mathrm{mg}$ as this drug reaches peak concentration in $2 \mathrm{~h}$ after administration. Doxycycline is one of the most effective oral antibiotics for inflammatory acne and therefore is used to effectively inhibit the growth of $P$. acne [7]. The use of oral antibiotics is generally indicated for moderate to severe inflammatory acne, or those who are resistant to previous topical therapy. Oral antibiotics such as macrolides, tetracyclines (doxycycline, minocycline, and lymecycline) are often used. These antibiotics are agents that inhibit the growth of $P$. acne in addition to their anti-inflammatory property. Tetracyclines are used frequently because due to the low-cost and high efficacy. Doxycycline and minocycline are preferred because of their low gastrointestinal irritation effect, solubility in fats, and better penetration of the pilosebaceous follicles. In addition, the resistance rate of $P$. acne to this class was less frequently reported compared to macrolides.

Some unique ingredients of serum include growth factor, platelet-rich plasma (PRP), Vitamin A, and many more. As we know that topical acne vulgaris treatment uses topical Vitamin A derivatives (tretinoin) which works by its anti-inflammatory, suppression of sebum production, and increases skin turnover [8]. In this study, the measurement of serum Vitamin A content 


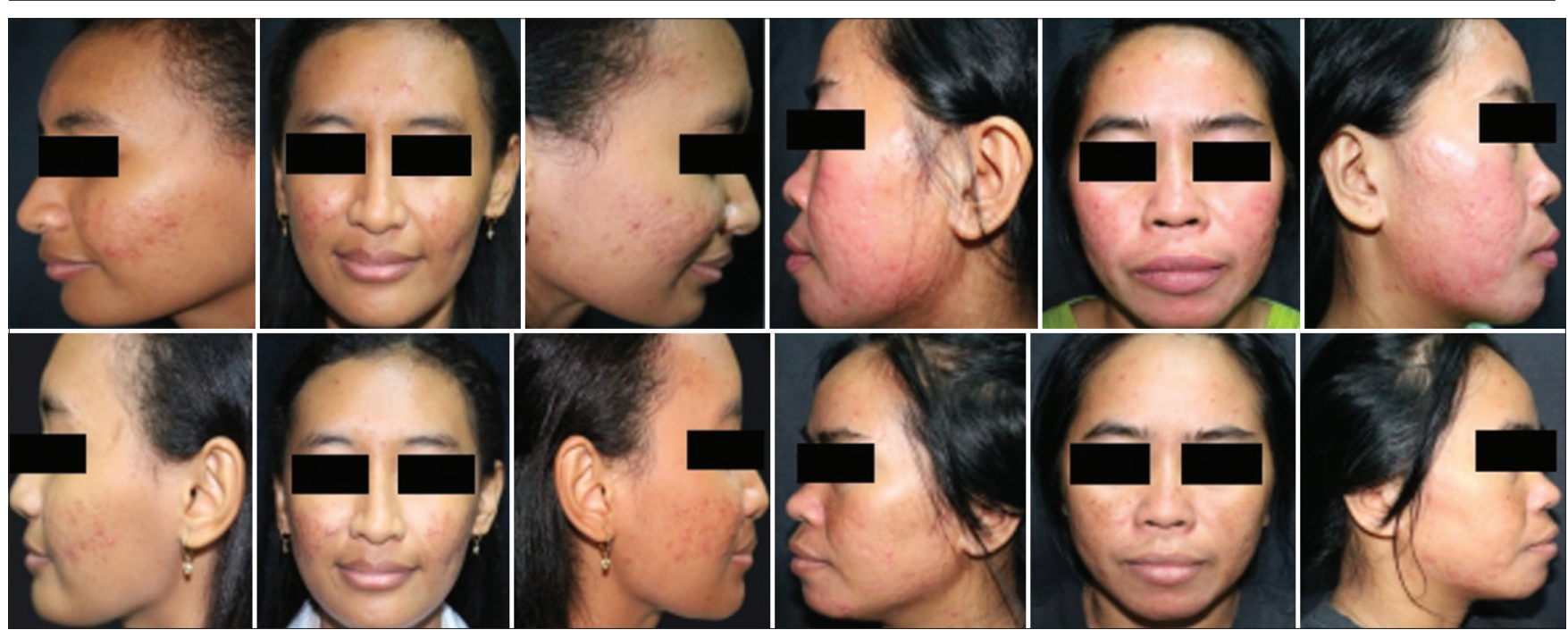

Figure 1: Clinical improvement after 28 days of the autologous serum only group

Table 2: Post-treatment result of the autologous serum and single $200 \mathrm{mg}$ doxycycline

\begin{tabular}{lll}
\hline Variable & D1-D28 & \\
\cline { 2 - 3 } & Median & p-value \\
\hline Decrease in number of blackheads (\%) & $98(18.8)$ & $<0.001$ \\
Decrease in number of papules (\%) & $37(26.6)$ & $<0.001$ \\
Decrease in number of pustules $(\%)$ & $18(56.0)$ & $<0.001$ \\
\hline${ }^{*}$ Significant if $p<0.05$ (Wilcoxon test). & &
\end{tabular}

was done using Enzyme-Linked Immunosorbent Assay test.

Kinda et al. explain that Vitamin A (All-trans retinoic acid [ATRA]) and Vitamin $D(1,25 D 3)$ share the retinoid $X$ receptor as a common receptor that encodes and functions to inhibit Th17. ATRA and 1,25D3 are generally used for therapy by dermatologists because they have the ability to modulate the immune response [9].
In this study, menstrual estradiol levels (follicular phase) were obtained in levels ranging from 20.8 to $200.5 \mathrm{pg} / \mathrm{mL}$. The concentration of estradiol levels is lowest during the menstrual phase with serum concentrations around 40-200 pg/mL. Meanwhile, the level of estradiol during preovulation was around 250-500 pg/mL, so it was concluded that the estrogen levels in the menstrual phase were lower than that of the preovulatory phase. Low estrogen levels are associated with the severity of acne. Androgen hormones increase the production of sebum, so that the sebum levels in patients with acne vulgaris are higher than in normal people [10]. There is a relationship between sebum excretion and the severity of acne vulgaris because the sebum levels in patients with severe acne vulgaris are found to be higher in levels. Estradiol can inhibit sebum production to reduce sebum levels. The use of
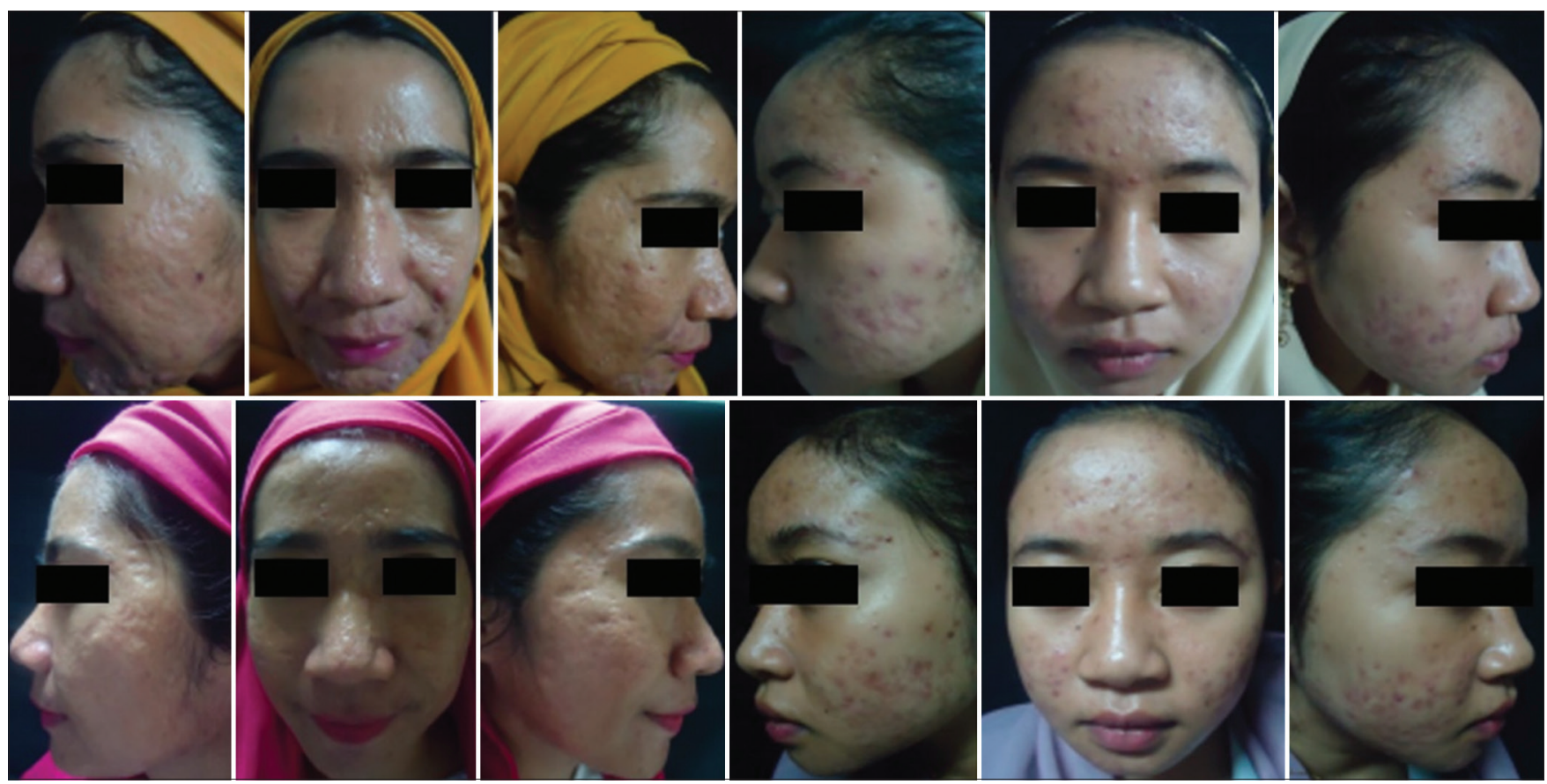

Figure 2: Clinical improvement after 28 days of the autologous serum and doxycycline group 
oral contraceptives for the treatment of acne vulgaris contains estradiol [11]

PRP works through alpha-granule degranulation in platelets containing growth factors. It is ideal to collect the PRP blood in a tube containing sodium citrate as an anticoagulant to keep growth factor active. Biologically active cellular components such as growth factors are preserved, when the platelets are held in an inactive state so that premature degranulation does not occur; therefore, the cellular components are held in a container that can protect the platelets. After platelet activation, the granules then fuse into the cell membrane by a degranulation process, activating growth factors, which bind to target cell transmembrane receptors, such as mesenchymal stem cells, fibroblasts, endothelial cells, and epidermal cells. This binding activates intracellular signaling proteins that express gene sequences resulting in cell proliferation, collagen synthesis, cell extracellular formation, and many other pathways to promote healing and repair processes. Platelets are damaged with degraded cellular components or are unable to induce this response [12], [13].

Based on this theory, PRP can be used for skin rejuvenation because it can activate growth factors that result in cell proliferation and stimulate collagen synthesis which can lead to healing and repair processes. In addition, PRP is reported to be associated with collagen expression, hyaluronic acid production, and fibroblast proliferation which is thought to be mediated by insulin-like growth factor, epidermal growth factor which binds to the outer surface of the fibroblast cell membrane activates signal proteins to realize extracellular matrix synthesis and collagen repackaging. Transforming Growth Factor b1 and platelet-derived growth factor are able to increase hyaluronic acid synthesis by increasing regulation of hyaluronic synthase expression. Hyaluronic acid can attract water into the space between cells and retain intracellular water so to maintain skin elasticity [13], [14], [15].

The improvement in acne lesions in this study was thought to be due to the involvement of growth factors, PRP, Vitamin A, and estradiol. Vitamin A works as an anti-inflammatory reduces sebum production, and increases skin turnover, estradiol works to inhibit androgens so that it can control reducing sebum production, growth factor works as an anti-inflammatory. Based on these contents, it is assumed that in the autologous serum that works together according to the pathogenesis of acne vulgaris.

Although the decrease in lesion number was more significant in the combination group, a head-tohead comparison was unable to be done due to the limited number of subjects in each group. Future studies with larger number of participants should be conducted to confirm this result.

\section{Conclusion}

Autologous serum contains many components including growth factors, PRP, Vitamin $A$, and estradiol which work synergistically to inhibit the pathomechanism of acne vulgaris. Addition of doxycycline $200 \mathrm{mg}$ orally as a single dose functions as an anti-inflammatory agent and was effective in improving acne lesions.

\section{References}

1. Engler D. Acne. S Afr Pharm J. 2016;83(10):27-34

2. Fox L, Csongradi C, Aucamp M, du Plessis J, Gerber M Treatment modalities for acne. Molecules. 2016;21(8):1063. http://doi.org/10.3390/molecules21081063 PMid:27529209

3. Bhat YJ, Latief I, Hassan I. Update on etiopathogenesis and treatment of Acne. Indian J Dermatol Venereol Leprol. 2017;83(3):298-306. http://doi.org/10.4103/0378-6323.199581 PMid:28195079

4. Thielitz A, Krautheim A, Gollnick $H$. Update in retinoid therapy of acne. Dermatol Ther. 2006;19(5):272-9. http://doi. org/10.1111/j.1529-8019.2006.00084.x PMid:17014482

5. Lynn DD, Umari T, Dunnick CA, Dellavalle RP. The epidemiology of acne vulgaris in late adolescence. Adolesc Health Med Ther 2016;7:13-25. http://doi.org/10.2147/AHMT.S55832 PMid:26955297

6. Pugashetti R, Shinkai K. Treatment of acne vulgaris in pregnant patients. Dermatol Ther. 2013;26(4):302-11. http://doi. org/10.1007/s40265-013-0060-0 PMid:23657872

7. Tan AU, Schlosser BJ, Paller AS. A review of diagnosis and treatment of acne in adult female patients. Int $\mathrm{J}$ Womens Dermatol. 2018;4(2):56-71. http://doi.org/10.1016/j. ijwd.2017.10.006 PMid:29872679

8. Langer C, Mahajan V. Platelet-rich plasma in dermatology. J K Sci. 2014;16(4):147-50.

9. Kelhälä $\mathrm{HL}$, Palatsi R, Fyhrquist N, Lehtimäki $\mathrm{S}$, Väyrynen JP, Kallioinen M, et al. IL-17/Th17 pathway is activated in acne lesions. PLoS One. 2014;9(8):e105238. http://doi.org/10.1371/ journal.pone. 0105238 PMid:25153527

10. Shaw JC. Acne: Effect of hormones on pathogenesis and management. Am J Clin Dermatol. 2002;3(8):571-8. http://doi. org/10.2165/00128071-200203080-00007 PMid: 12358558

11. Sexton $S$. Acne vulgaris: Treatment guidelines from the AAD. J Am Acad Dermatol. 2016;74(5):945-73.

12. Kumar S, Mahajan BB, Kaur S, Singh A. Autologous therapies in dermatology. J Clin Aesthet Dermatol. 2014;7(12):38-45 PMid:25584137

13. Du R, Lei T. Effects of autologous platelet-rich plasma injections on facial skin rejuvenation. Exp Ther Med. 2020;19(4):3024-30. http://doi.org/10.3892/etm.2020.8531 
PMid:32256789

14. Draelos ZD, Rheins LA, Wootten S, Kellar RS, Diller RB. Pilo study: Autologous platelet-rich plasma used in a topical cream for facial rejuvenation. J Cosmet Dermatol. 2019;18(5):1348-52. http://doi.org/10.1111/jocd.13088

PMid:31350798
15. Amable PR, Carias RB, Teixeira MV, da Cruz Pacheco I, Corrêa do Amaral RJ, Granjeiro JM, et al. Platelet-rich plasma preparation for regenerative medicine: optimization and quantification of cytokines and growth factors. Stem Cell Res Ther. 2013;4(3):67. http://doi.org/10.1186/scrt218 PMid:23759113 